\title{
Micromagnetism and magnetization reversal of micron-scale (110) Fe thin-film magnetic elements
}

\author{
J. Yu, U. Rüdiger, and A. D. Kent* \\ Department of Physics, New York University, 4 Washington Place, New York, New York 10003 \\ L. Thomas and S. S. P. Parkin \\ IBM Research Division, Almaden Research Center, San Jose, California 95120
}

\begin{abstract}
Magnetic force microscope (MFM) imaging in conjunction with longitudinal Kerr hysteresis loop measurements have been used to investigate the micromagnetic behavior of micron scale epitaxial (110) bcc $\mathrm{Fe}$ thin-film elements (50-nm thick) with rectangular, triangular, and needle-shaped ends and competing magnetic anisotropies. Thin-film elements of $2-\mu \mathrm{m}$ width and $6-\mu \mathrm{m}$ length and greater have been fabricated with their long axis oriented either parallel or perpendicular to the [001] in-plane magnetocrystalline easy axis. For elements with their long axis perpendicular to the [001] direction, the end shape is critical in determining domain nucleation, domain configurations, and magnetic hysteresis. The magnetization reversal mechanisms are revealed by direct field dependent MFM imaging. Magnetic vortex configurations within elements during reversal are seen to be affected by small changes in element corner shape. Similarly, small trapped domains and domain walls are observed to applied fields significantly larger than the coercive field and apparent magnetic saturation field, as determined by hysteresis loop measurements of arrays of elements. These are shown to have a dramatic effect on the character of the low-field magnetic hysteresis. Particles with long axis parallel to the [001] direction have large remanence and switching fields which also depend sensitively on end shape. The angular dependence of the switching field observed in these elements is contrasted to that of magnetization reversal by coherent rotation.
\end{abstract}

\section{INTRODUCTION}

Micron scale patterned thin-film ferromagnetic elements are a topic of great interest ${ }^{1-3}$ with important applications to magnetic information storage technology. ${ }^{4,5}$ Magnetic behavior such as hysteresis, magnetization reversal, and domain configurations change significantly from that in extended thin films due to the presence of lithographically defined boundaries. ${ }^{1-3}$ For in-plane magnetized films, these boundaries increase the importance of dipolar or shape anisotropy, as a normal component of the magnetization at the boundary leads to magnetic charges. ${ }^{6}$ The magnetic properties are in general a consequence of the interplay between magnetostatic, exchange, magnetocrystalline, and Zeeman energies. Thermal fluctuations may also play an important role in the magnetization reversal, and become particularly important in nanometer scale ferromagnetic structures. While at micron scale sizes individual elements have only a small number of domains, ${ }^{1-3}$ the magnetic behavior is still in general quite complex and challenging to model using either analytic or numerical micromagnetic techniques. ${ }^{1,7,8}$ An understanding of magnetic phenomena in micron scale elements is nonetheless essential to applications of these materials in magnetoelectronics and experiment can also guide micromagnetic modeling. As we show, micron scale materials with wellcharacterized magnetic interactions can provide important insight into the control of magnetic characteristics by shape and the effects of magnetic topology on magnetization reversal.

We have examined the physical consequences of element shape and anisotropy on magnetic phenomena in this size range by studying model thin-film microstructures. Epitaxial growth and advanced microfabrication techniques have been used to produce elements with controlled magnetocrystalline anisotropy and shape. Magnetization reversal and domain configurations are studied in individual magnetic elements by direct imaging of domains in applied magnetic fields with a magnetic force microscope (MFM). Magnetic hysteresis is studied in arrays of nominally identical elements using longitudinal Kerr hysteresis loop measurements (MOKE). This combination enables an understanding of the connection between local magnetic domain configurations within elements and magnetic hysteresis of an array of such elements (such as the coercivity, remanent magnetization, and switching characteristics).

In this paper, we present results of a study of (110) bcc Fe thin-film patterned elements with different end shapes and in which the elements' long axis has been oriented with respect to the in-plane crystallographic directions. Film demagnetization factors confine the magnetization to the (110) plane. This plane has a strong uniaxial component to the magnetic anisotropy, with [001] magnetic easy axis, and a smaller cubic magnetic anisotropy, with in-plane [1 $1 \overline{1}]$ hard and [1] 10$]$ medium magnetic axis. Elements with two different orientations have been studied; (i) with the element long axis perpendicular to the in-plane magnetocrystalline easy [001] axis direction and (ii) with the long axis parallel to the easy magnetic axis. In the former case a competition between magnetic energies results, whereas in the latter case the magnetocrystalline energy reinforces the element shape anisotropy. In both cases, particles of different end shape have 


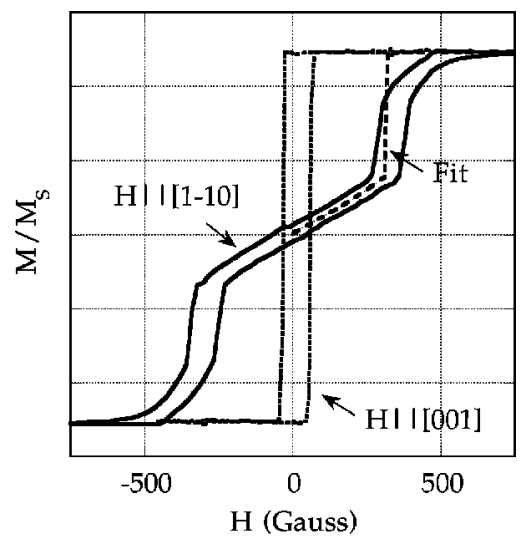

FIG. 1. Longitudinal Kerr hysteresis loop measurements on a 50-nm-thick epitaxial (110) Fe film with the applied field parallel to the [001] direction (easy magnetocrystalline axis) and parallel to the [1 $\overline{1} 0]$ direction (hard axis). Also included is the fit (dashed line) of the hard axis hysteresis loop.

been studied, consisting of square ends (or rectangular particles), triangular and needle-shaped ends. While studies of (100) Fe thin-film elements with fourfold in-plane anisotropy have been reported, ${ }^{1}$ we know of no studies of micron scale (110) plane Fe structures in which the role of end shape has been systematically examined.

\section{FABRICATION AND CHARACTERIZATION}

(110) oriented 50-nm-thick Fe films were grown on $a$-axis $(11 \overline{2} 0)$ sapphire substrates using UHV $e$-beam evaporation techniques. First, a 10-nm-thick Mo seed layer was deposited at $900 \mathrm{~K}$ followed by a 50 -nm-thick Fe layer at $510 \mathrm{~K} .{ }^{9,10}$ The deposition rates for the Mo seed layer and the Fe layer were $0.2 \mathrm{~nm} / \mathrm{s}$ and $0.5 \mathrm{~nm} / \mathrm{s}$, respectively. The films were protected against corrosion with a 5-nm-thick Mo capping layer. X-ray $\theta / 2 \theta$ scans reveal a complete (110) film orientation and $\mathrm{x}$-ray pole figures show that the $\mathrm{Fe}$ in-plane [1 $\overline{1} 1]$ axis is parallel to [0001] direction of the sapphire substrates. An anisotropic in-plane strain in these films induces an inplane uniaxial magnetic anisotropy with the easy axis along the [001] direction in addition to the cubic anisotropy, characteristic of bulk bcc Fe. ${ }^{10}$ Longitudinal hysteresis loop measurements on an unpatterned (110) Fe film with the applied magnetic field parallel to the $[1 \overline{1} 0]$ are consistent with two and fourfold in-plane components of the magnetic anisotropy. Both the easy $(H \|[001])$ and the hard $(H \|[1 \overline{1} 0])$ axis Kerr loop are shown in Fig. 1. The hard axis loop (solid line) of a 50-nm-thick (110) Fe film has been modeled using the in-plane anisotropy energy density:

$$
E=\left(K_{1}+K_{u}\right) \sin ^{2} \theta-\frac{3}{4} K_{1} \sin ^{4} \theta
$$

where $K_{1}$ is the cubic anisotropy constant, $K_{u}$ is the uniaxial anisotropy constant, and $\theta$ is the angle between the magnetization and the [001] axis. From this fit the cubic and the uniaxial anisotropy constants have been determined to be $K_{1}=6.3 \times 10^{5} \mathrm{erg} / \mathrm{cm}^{3}$ and $K_{u}=3.0 \times 10^{5} \mathrm{erg} / \mathrm{cm}^{3}$. There is some deviation of this fit from the data near magnetic satu-

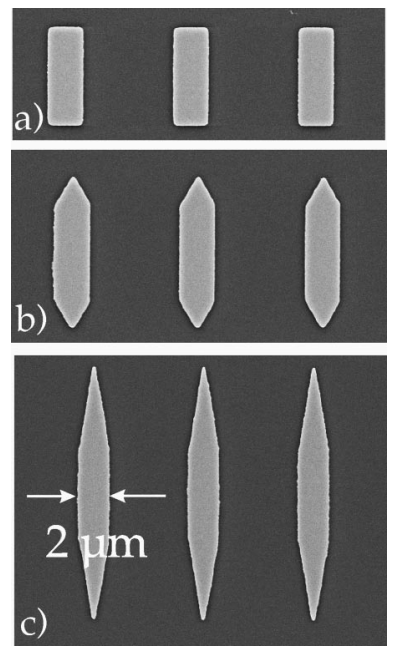

FIG. 2. SEM images of (a) rectangular, (b) triangular, and needle-shaped 2- $\mu \mathrm{m}$ wide (110) Fe particles.

ration. This is likely due to small variations in magnetic anisotropy in the film, and particularly the presence of regions of enhanced anisotropy.

These $\mathrm{Fe}$ films have been patterned into particle arrays with rectangular, triangular, and needle-shaped ends, using electron-beam lithography and Argon ion milling. The magnetic properties and the magnetization reversal process of arrays of particles were studied via magneto-optic Kerr effect (MOKE) hysteresis loops and individual particles were studied with a MFM which incorporated an in situ electromagnet. The latter enabled fields up to 1500 Oe to be applied during imaging experiments. Here we report results on $2-\mu \mathrm{m}$-wide particles. Figure 2 shows scanning electron microscope (SEM) images of these particles with (a) rectangular, (b) triangular, and (c) needle-shaped ends. The rectangular particles have a length-to-width ratio of $3: 1$, while the other two particle types have two additional triangular or needlelike ends, which makes these particles greater in net "tip-to-tip" length. For example, the overall length of the needle is nine times its width. All particles are separated by four times their width and three times their length, so that dipolar interactions between neighboring particles within the array are negligible. ${ }^{11}$

\section{RESULTS AND DISCUSSION}

\section{A. Magnetic easy axis perpendicular to the long particle axis}

First we consider the case in which the magnetic easy axis is perpendicular to the long axis of the particle, for which a competition between magnetic energies occurs. Figure 3 shows the hysteresis loops of rectangular and needlelike end particles in longitudinal and transverse field geometries. $\mathrm{H}$-longitudinal refers to the applied magnetic field parallel to the long axis of the particle while H-transverse refers to the field perpendicular to the long axis (i.e., $H \|[001]$ ).

In the longitudinal case [Fig. 3(a)] the hysteresis loops of rectangular and needlelike end particles both exhibit a small remanence $\left(\approx 0.09 M_{s}\right)$ at $H=0$. For the rectangular particles the saturation field $H_{S}$ and the nucleation field $H_{N}$ are $H_{S}=400$ Oe and $H_{N}=300$ Oe, respectively. For needlelike particles $H_{S}$ is $300 \mathrm{Oe}$ and $H_{N}$ is $200 \mathrm{Oe}$. 


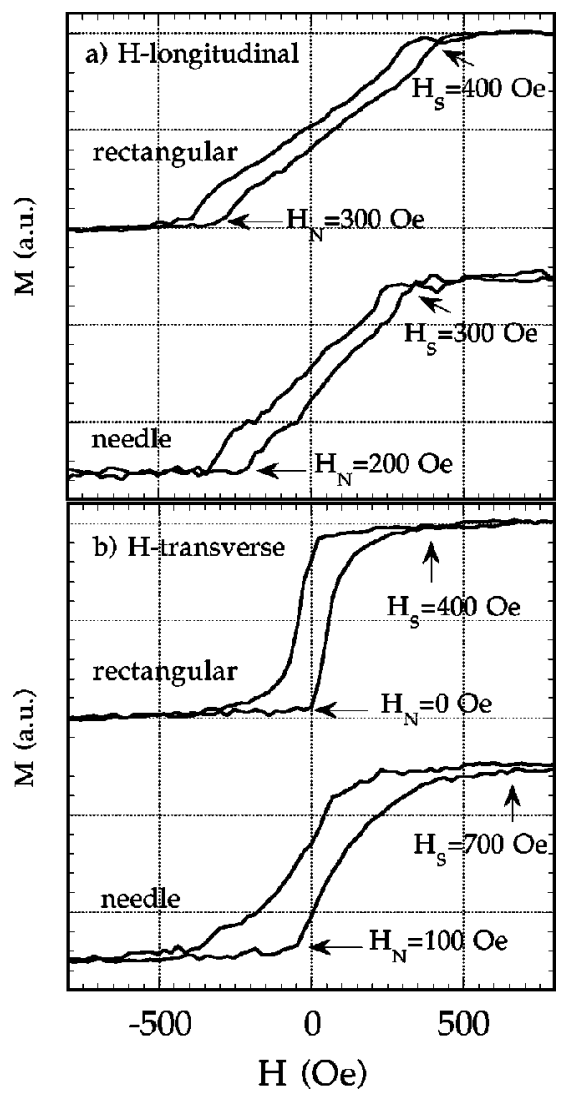

FIG. 3. Longitudinal MOKE hysteresis loop measurements on rectangular and needle-shaped (110) Fe particles with the applied magnetic field (a) parallel (H-longitudinal) and (b) perpendicular (H-transverse) to the long axis of the particles.

In the transverse case [see Fig. 3(b)] the rectangular particles show nearly full remanence, a nucleation field of approximately $H_{N}=0 \mathrm{Oe}$, and magnetic saturation field of $H_{S}=400$ Oe. In contrast, the needle-shaped particles show a smaller remanence and a nucleation field of $H_{N}=100 \mathrm{Oe}$ and a saturation field of $H_{S}=700$ Oe.

MFM imaging has been performed in order to correlate these magnetic hysteresis loops with the magnetization reversal processes and magnetic domain patterns of individual particles. The influence of the particles shape and the magnetic history on the nucleation process of magnetic domains and on the magnetic domain configurations at $H=0$ has been studied. A majority $(\sim 80 \%)$ of the particles within an array show the same domain structure in zero field. ${ }^{12}$ A few particles within this majority are selected for detailed field dependent studies of magnetization reversal. The NiFe coated MFM tips used are magnetized vertically prior to imaging studies. ${ }^{13}$ Images typically highlight magnetic poles at the boundaries of the particles and magnetic charges associated with domains walls.

Figure 4 presents MFM images of the magnetic domain configurations of rectangular and needle-shaped particles at selected magnetic fields in the longitudinal field geometry. In the case of the rectangular particle [Figs. 4(a)-(e)], domains are visible first at the element corners at an applied field of approximately 420 Oe. Figure 4(a) reveals a magnetization pattern with domain walls at the particles ends and edge domains. In this particular element, both edge domains are

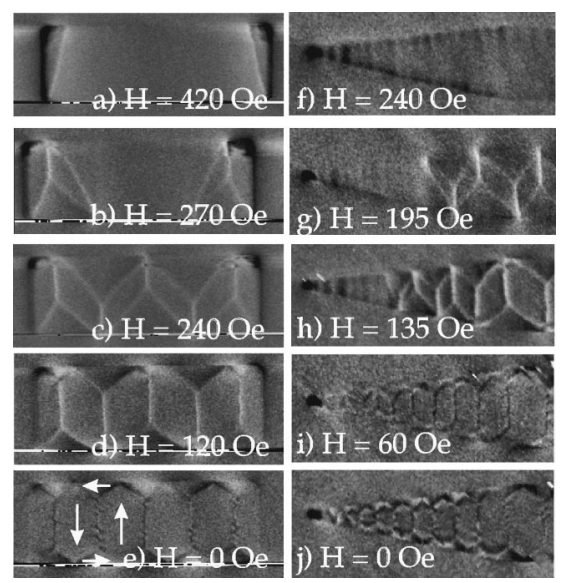

FIG. 4. Field dependent MFM images on (a)-(e) rectangular and (f)-(j) needle-shaped (110) Fe particles in the longitudinal field geometry.

larger on the top edge of the particle, which implies that the magnetization at the left and right edges are in opposite directions. This is sometimes called a " $\mathrm{C}$ " magnetic state. ${ }^{14}$ Other particles have shown edge domains with long edges on opposite sides of the particles, ' $N$ ' states. On reduction of the longitudinal applied field [Fig. 4(b)] a vortex is formed and domains move towards the center of the particles. The arrows in Fig. 4(e) indicate the in-plane magnetization direction of the stripe domains with the magnetization parallel or antiparallel to the easy magnetocrystalline axis and the flux closure domains where the magnetization is directed perpendicular to the easy axis. The MFM image of the demagnetized state at $H=0$ [Fig. 4(e)] is consistent with the small remanence seen in MOKE hysteresis loops [see Fig. 3(a)]. In contrast, for the needle-shaped particles the nucleation of magnetic domains occurs at lower magnetic field $(\approx 195$ Oe $)$ and closer to the particle center. In the demagnetized state at $H=0$ the width $d$ of the stripe domains in the center of the needle-shaped particle [see Fig. 4(j), right side] is significantly smaller than that observed in the rectangular particle [Fig. 4(e)]. The value of the domain width near the center of the elements is determined to be $d_{N}=0.7 \mu \mathrm{m}$ and $d_{R}=1.0 \mu \mathrm{m}$ for the needle-shaped and the rectangular particle, respectively.

The MFM images in Figs. 5 and 6 give an overview of the nucleation and motion of magnetic domains in the presence of a transverse applied magnetic field. For both shapes, images are shown on decreasing the magnetic field from the magnetic saturation field. Figure 5 shows the evolution of magnetic domains for a rectangular particle in the transverse field geometry. Starting from a positive magnetic field domains first appear close to the particle corners but, as discussed in detail in a following section, the location of these domains also depends strongly on the magnetic history. The hysteresis loop in the transverse field geometry in Fig. 3(b) indicates that the particle is almost fully saturated above 400 Oe, while the MFM images in Figs. 5(a) and (j) show very small domains along the long edges of the particle. For increasing negative fields, where two domain walls approach each other [see Figs. 5(g) to (j)], a field much larger than the coercive field is necessary to annihilate the domain walls and reversed magnetic domains near the center of the particle. 


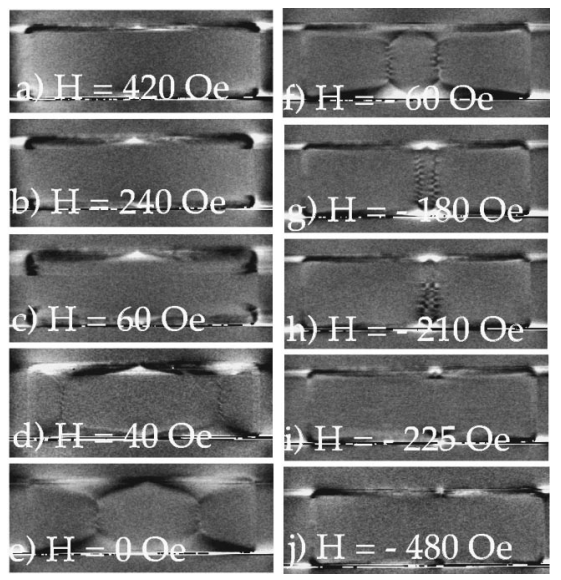

FIG. 5. Field dependent MFM images of the magnetic domain configuration in a rectangular particle in transverse field geometry.

For saturation fields higher than approximately 500 Oe the nucleation occurs at fields of only 40-60 Oe [see Figs. 5(c) and (d)]. This is not consistent with the magnetic hysteresis measurements, as the hysteresis loop [Fig. 3(b)] indicates almost full remanence while the MFM image in Fig. 5 shows a more or less demagnetized state. The stray field of the MFM tip may be influencing the nucleation process. Likely the stray field has initiated the nucleation of domains and therefore shifted the nucleation field to higher values in comparison with that determined via MOKE measurements. The domain structure observed in zero field does not appear to be affected by the MFM tip, as the images are both stable in time and observed on a number of different particles after the same magnetic preparation. Note that in this geometry the stripe domain width is $d_{R}=2.8 \mu \mathrm{m}$ in zero field and is larger than that in the longitudinal field geometry.

For the needle-shaped particle (see Fig. 6) the nucleation of domains takes place first at the outermost part of the needle which is in clear contrast to the longitudinal field geometry and occurs in a field range of 100-200 Oe. Like the rectangular particles, the average domain width of $d_{N}$ $=1.9 \mu \mathrm{m}$ in the center of the particle is clearly increased in comparison with the longitudinal field geometry.

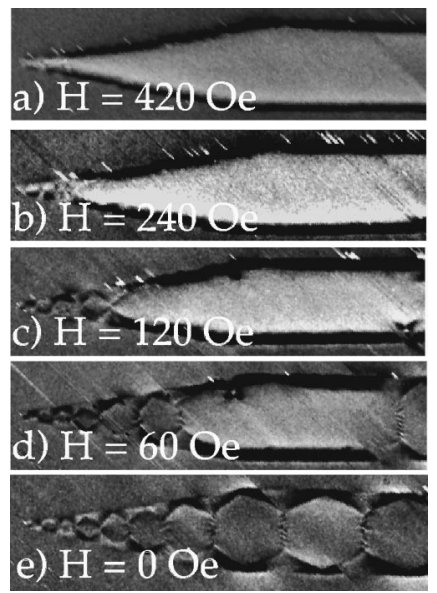

FIG. 6. Field dependent MFM images of the magnetic domain configuration in a needle-shaped particle in transverse field geometry.
The interplay between exchange, Zeeman, shape, and magnetocrystalline anisotropy energies determines the micromagnetic structure and magnetization reversal process. With decreasing particle size, the demagnetizing field arising from the magnetic charges formed at particle edges becomes important. This introduces a shape anisotropy with the easy axis parallel to the long axis of the particle, and perpendicular to the magnetocrystalline easy axis. In such a case, it is well known that for large enough magnetocrystalline anisotropy, a stripe domain configuration minimizes the free energy. ${ }^{7,8}$ If this magnetic anisotropy energy is sufficiently large to favor such a state, yet still much smaller than the demagnetization energy, stripe domains in conjunction with flux closure domains at $H=0$ are favored. This is the case for our Fe microstructures for which the ratio of magnetocrystalline anisotropy to magnetostatic energy is small $\left(K \ll M^{2}\right)$. Such stripe domain configurations with flux closure domains have also been observed in microfabricated $\mathrm{Fe}$ wires $^{9,15}$ and submicron $\mathrm{Fe}$ thin-film elements. ${ }^{12}$

In the transverse field geometry, both for rectangular and needle-shaped particles, domains nucleate near the particle ends on reducing the applied field from its saturation value. The demagnetizing fields generated when the magnetization is rotated away from the long axis of the particle are much larger at the outermost end of the needle-shaped particles than at the corners of the rectangular particles. Therefore higher nucleation and saturation fields are observed for the needlelike particles. After nucleation of magnetic domains at the ends of the particle, on further reduction of the applied field, the domains move towards the particle center forming a flux closure multidomain state at $H=0$.

For the longitudinal field geometry both the shape anisotropy and Zeeman energy favor a magnetization parallel to the applied field. The enhanced shape anisotropy at the outermost part of the needles now favors an alignment of the magnetization parallel to the particles long axis. Hence the nucleation and saturation fields for the needlelike particles are slightly lower than those observed for the rectangular particles. The shape anisotropy has a more remarkable impact on the location where the magnetic domains nucleate first. In the case of the needle-shaped particle the enhanced shape anisotropy near the long tail of the needle suppresses the formation of domains at the end of the needle.

Small changes in corner shape affect the magnetization topology during reversal. Figure 7 shows MFM images of (a) a rectangular particle, (b) a rectangular particle with a cut corner on the lower left-hand side, and (c) with cut corners on the lower left- as well as the upper right-hand side. All MFM images are performed in a longitudinal oriented field of 300 Oe which is just below the nucleation field. For identification, the approximate magnetization direction of the domains are labeled with arrows. At both sides of all three different particles a magnetic vortex configuration is visible. A magnetic vortex configuration at the corners of the particles leads to a minimization of the magnetostatic energy at the cost of exchange and magnetocrystalline energy. The rectangular particle in Fig. 7(a) shows a vortex with righthand chirality on both ends and therefore both vortex centers are located on the same long edge of the particle. The MFM images in Figs. 7(b) and (c) demonstrates how sensitive the symmetry of the vortex state is to slight changes of the par- 

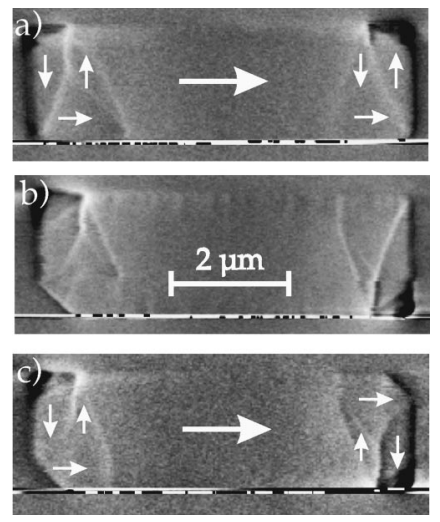

FIG. 7. MFM images of magnetic vortices in (a) a rectangular particle, (b) a rectangular particle with one cut corner, and (c) a particle with two cut corners at an applied field of $H=300$ Oe. The arrows illustrate schematically the circulation of the magnetic vortices at the element edges.

ticle geometry. By cutting one corner Fig. 7(b) or two corners on opposite sites, the center of the vortices appears on the side with the remaining rectangular corner geometry. In both of these cases the vortices in each particle are of opposite chirality.

In the transverse field geometry the topology of the magnetization during reversal has an important influence on hysteresis. Figure 8 shows normalized hysteresis loops in transverse field geometry for an array of rectangular particles as function of a maximum applied field $H_{\max }$, which has been varied from 212-997 Oe. The arrow in Fig. 8 is a guide to how the shape of the hysteresis loop is changed at low field as a function of $H_{\max }$. For small $H_{\max }(<300 \mathrm{Oe})$, a continuous decrease of the magnetization near zero field is visible. In contrast, for larger $H_{\max }(>300 \mathrm{Oe})$ the hysteresis loops show a sharp drop near $H=0$ Oe, indicating an abrupt nucleation or growth of reversed magnetization. In Fig. 9 the normalized magnetization of the array is plotted at applied fields of $50 \mathrm{Oe}, 75 \mathrm{Oe}$, and $100 \mathrm{Oe}$ as a function of $H_{\max }$. For $H_{\max }$ smaller than $300 \mathrm{Oe}$, at all three field values investigated, a drop of up to $25 \%$ of the saturation magnetization has been observed, whereas for $H_{\max }$ larger than approximately 300 Oe only a small $(10-15 \%)$ deviation of the

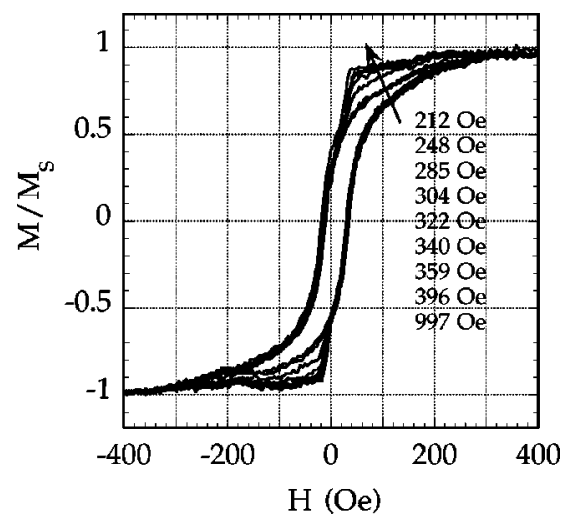

FIG. 8. Normalized hysteresis loops (MOKE) of an array of rectangular particles in the transverse field geometry where the maximum applied magnetic field $H_{\max }$ has been varied. The arrow indicates the evolution of the shape of the hysteresis loop with increasing maximum applied field $H_{\max }$.

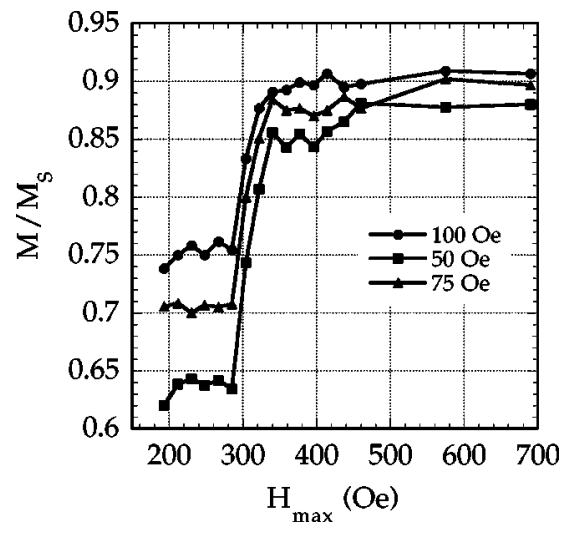

FIG. 9. Variation of the normalized magnetization at 50, 75, and $100 \mathrm{Oe}$ as a function of the maximum applied field $H_{\text {max }}$.

magnetization from full saturation is measured. All curves show a transition at around $H_{\max }=300$ Oe. This transition marks a fundamental change in the magnetization reversal process from a continuous magnetization reversal process $\left(H_{\max }<300 \mathrm{Oe}\right)$ consisting of domain-wall motion, to one $\left(H_{\max }>300 \mathrm{Oe}\right)$ driven by a sudden nucleation or growth of reversed domains in the range of $40 \mathrm{Oe}$.

MFM images show that, for applied fields smaller than 300 Oe, small domains remain close to the long edges of the particle and expand during the reversal process. The MFM images in Figs. 10(a) at 100 Oe and (b) at 50 Oe were both taken after first applying a transverse magnetic field of 900 Oe. These images indicate the presence of small reversed domains. With decreasing applied field these small domains become slightly larger. However, at these fields the amount of reversed magnetization is negligible in comparison with that parallel to the applied field, in accordance with the data in Fig. 9 with $H_{\max }$ larger than 350 Oe. MFM measurements were performed on the same particle after applying a maximum field of only 230 Oe. MFM images at 100 Oe [Fig. 10(c)] show that reversed domains are present on both long edges of the particle. At 50 Oe [Fig. 10(d)] a large reversed

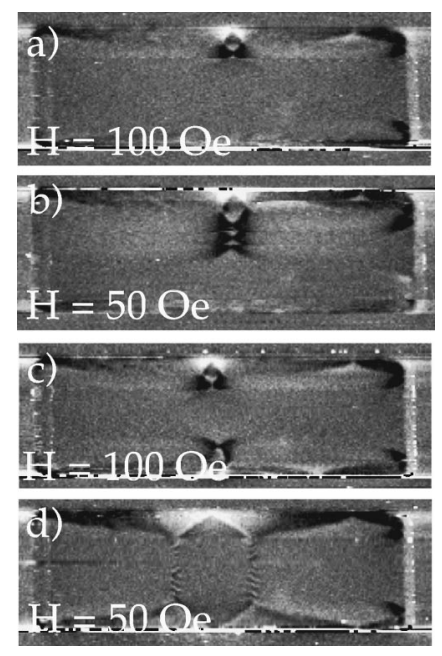

FIG. 10. MFM images of the nucleation process in a rectangular particle in transverse field geometry. The magnetic domain configuration at (a) $100 \mathrm{Oe}$ and (b) $50 \mathrm{Oe}$ are shown after applying a maximum field of $H_{\max }=900$ Oe as well as at (c) 100 Oe and (d) 50 Oe after applying a maximum field of $H_{\max }=230$ Oe. 
domain appears in conjunction with flux closure domains parallel to the long edges of the particle. These results suggest that pinning of the magnetization at the particle boundaries depends on the maximum applied transverse field and that this pinning affects the low-field magnetization reversal. This pinning may be associated with structural disorder at the particle boundaries caused by the ion-milling process used to form these structures. Within this scenario, large applied transverse fields more effectively pin domain walls close to the particle edges leading to the sudden magnetization jumps observed at low fields.

\section{B. Magnetic easy axis parallel to the long particle axis}

If the long axis of the particles is chosen to be parallel to the easy magnetocrystalline axis the particles with rectangular, triangular, and needlelike ends show a single domain state in zero applied field. Here the effective uniaxial anisotropy $K_{\text {eff }}$ results from both the magnetocrystalline and the shape anisotropy. In the following the $z$ axis is taken to be parallel to the long axis and the $x$ direction is taken to be perpendicular to the long axis of the particles. According to a coherent magnetization rotation model, the projection of the switching field onto the $x$ and $z$ axis is an asteroidlike curve. ${ }^{6,16}$ The effective uniaxial anisotropy energy density can be described via $E=K_{\text {eff }} \sin ^{2} \phi$ with the angle $\phi$ between the $z$ axis (particle's long axis) and the magnetization. Minimization of the system's free energy leads to the projection of the switching fields $H_{x}$ and $H_{z}$ in the $x-z$ plane described by

$$
\left(H_{x}\right)^{2 / 3}+\left(H_{z}\right)^{2 / 3}=\left(2 K_{e f f} / M\right)^{2 / 3} .
$$

Hysteresis loops have been performed on particle arrays with all three types of ends as function of the angle betweeen the particle long axis and the applied field, $\theta$. The insets of Fig. 11 show the hysteresis loops measured on an array of particles with needle-shaped ends at $\theta=0^{\circ}, \theta=50^{\circ}$, and $\theta$ $=90^{\circ}$. With increasing $\theta$ the hysteresis loops evolve gradually from a typical easy axis loop to a hard axis loop. From these kind of hysteresis loops the $H_{x}$ and $H_{z}$ values have been determined and plotted in Fig. 11 for particles with rectangular (solid rectangles), triangular (solid triangles), and needle-shaped (solid circles) ends. For the hard axis scan $\left(\theta=90^{\circ}\right)$ the $H_{x}$ values do not show a strong dependence on the exact shape of the particles. This is expected since the rotation of the magnetization will be largely uniform (coherent) across the particle, against magnetocrystalline and shape anisotropy factors. The $H_{x}$ values for rectangular and needlelike particles have been determined to be $H_{x, R}=780$ Oe and $H_{x, N}=890$ Oe, respectively. Magnetocrystalline anisotropy alone is expected to give an anisotropy field of $H_{a}=2\left(K_{u}\right.$ $\left.+1 / 4 K_{1}\right) / M=540$ Oe. The larger values observed are thus associated with the element shape anisotropy, which is largest for the needlelike particles. Using the measured $H_{x}$ values of rectangular and needle-shaped particles, asteroidlike curves for both particle types have been plotted. The dashed asteroidlike curve in Fig. 11 describes ideal coherent magnetization reversal in the rectangular particles and the dotted one is for needlelike particles. The strong deviations for $\theta$ $=0^{\circ}$ (easy axis loop) from the asteroidlike curves indicate, as expected, that the reversal is not coherent. In the case of

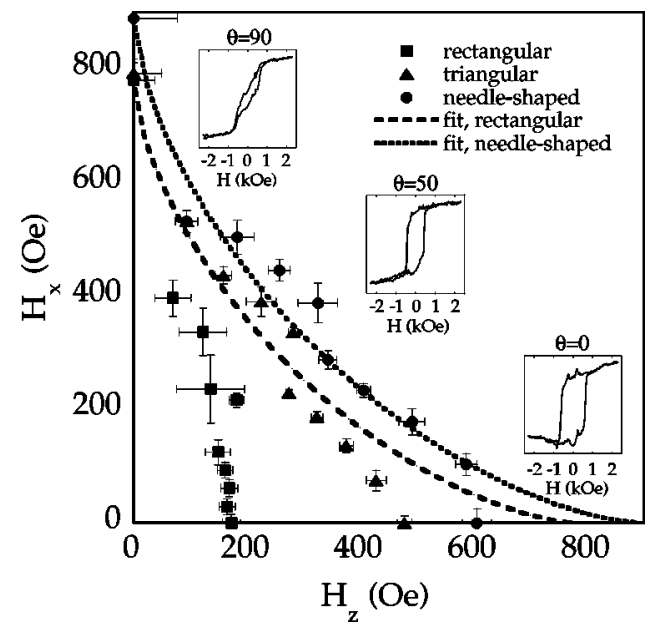

FIG. 11. Magnetization reversal on rectangular (solid rectangles), triangular (solid triangles), and needle-shaped (solid circles) particles with uniaxial magnetic anisotropy, i.e., the easy magnetocrystalline axis parallel to the long wire axis. $H_{z}$ and $H_{x}$ refer to the projection of the in-plane switching field parallel and perpendicular to the long wire axis, respectively. The insets show hysteresis loops as function of the magnetic field direction with respect to the long axis of the wire.

the rectangular and triangular particles, reversed domains likely nucleate at the particle ends, as we have observed for particles oriented perpendicular to the [001] axis. The reversal process is driven by this nucleation and subsequent domain wall movement. However, highly metastable magnetization states can be stabilized by blocking the nucleation of magnetic domains at the particle ends. This occurs in needlelike ended particles and results in behavior qualitatively similar to that predicted in a coherent rotation model. Again, this is due to the locally enhanced shape anisotropy at the outermost part of the needles which suppresses the nucleation of reversed domains. This result is rather remarkable in that large elements, with dimensions much larger than the exchange length (Fe $\left.\delta_{e x} \sim 20 \mathrm{~nm}\right)$, can show characteristics similar to nanometer scale single domain particles. This illustrates clearly the importance of end geometry on the reversal modes of micron scale thin-film elements. ${ }^{17,18}$

\section{SUMMARY}

In summary micron scale (110) Fe elements have been used as model materials to investigate the effect of shape and competing anisotropies on magnetization reversal and micromagnetic configurations. Direct imaging in applied fields with MFM combined with hysteresis loop measurements of arrays lead to a detailed understanding of the magnetization reversal. In samples with competing anisotropies, experiments illustrate the effect of element shape on the magnetization topology within elements and the effect of trappeddomains on low-field magnetic hysteresis. These results would clearly be interesting to simulate with fast numerical micromagnetic codes to provide a quantitative basis for understanding the observed magnetic properties in such materials.

\section{ACKNOWLEDGMENTS}

This research was supported by DARPA-ONR, Grant No. N00014-96-1-1207. Microstructures were prepared at the CNF, Project No. 588-96. 
*Author to whom correspondence should be addressed. Electronic address: andy.kent@nyu.edu

${ }^{1}$ E. Gu, E. Ahmad, C. Daboo, J. A. C. Bland, L. M. Brown, M. Rhrig, A. J. McGibbon, and J. N. Chapman, Phys. Rev. Lett. 78, 1158 (1997).

${ }^{2}$ M. Hehn, K. Ounadjela, J. P. Bucher, F. Rousseaux, D. Decanini, B. Bartenlian, and C. Chappert, Science 272, 1782 (1996).

${ }^{3}$ R. H. Koch, J. G. Deak, D. W. Abraham, P. L. Trouilloud, R. A. Altman, Y. Lu, W. J. Gallagher, R. E. Scheuerlein, K. P. Roche, and S. S. P. Parkin, Phys. Rev. Lett. 81, 4512 (1998).

${ }^{4}$ W. J. Gallagher, S. S. P. Parkin, Y. Lu, X. P. Bian, A. Marley, K. P. Roche, X. P. Altman, S. A. Rishton, C. Jahnes, T. M. Shaw, and G. Xiao, J. Appl. Phys. 81, 3741 (1997).

${ }^{5}$ E. D. Dahlberg and J. G. Zhu, Phys. Today 48 (4), 34 (1995).

${ }^{6}$ L. D. Landau, E. M. Lifshitz, and L. P. Pitaevskii, Electrodynamics of Continuous Media, 2nd ed. (Butterworth-Heinenann, Oxford, England, 1984).

${ }^{7}$ C. Kittel, Phys. Rev. 70, 965 (1946).

${ }^{8}$ S. Chikazumi, Physics of Ferromagnetism (Clarendon Press, Oxford, 1997).

${ }^{9}$ A. D. Kent, U. Rüdiger, J. Yu, S. Zhang, P. M. Levy, and S. S. P. Parkin, IEEE Trans. Magn. 34, 900 (1998).
${ }^{10}$ B. M. Clemens, R. Osgood, A. P. Payne, B. M. Lairson, S. Brennan, R. L. White, and W. D. Nix, J. Magn. Magn. Mater. 121, 37 (1993).

${ }^{11}$ M. P. Pardavi-Horvath, G. Zheng, G. Vertesy, and A. Magni, IEEE Trans. Magn. 32, 4469 (1996).

${ }^{12}$ J. Yu, U. Rüdiger, A. D. Kent, L. Thomas, and S. S. P. Parkin, J. Appl. Phys. 85, 5501 (1999).

${ }^{13}$ While NiFe is magnetically soft, we see no significant changes in MFM images with in-plane applied fields of up to 1000 Oe. Likely, magnetic shape anisotropy stabilizes the vertical tip magnetization.

${ }^{14}$ J. Shi, T. Zhu, U. Durlam, E. Chen, S. Tehrani, Y. F. Zheng, and J.-G. Zhu, IEEE Trans. Magn. 34, 997 (1998).

${ }^{15}$ U. Rüdiger, J. Yu, A. D. Kent, and S. S. P. Parkin, Appl. Phys. Lett. 73, 1298 (1998).

${ }^{16}$ E. C. Stoner and E. P. Wohlfarth, Philos. Trans. R. Soc. London, Ser. A 320, 599 (1948).

${ }^{17}$ T. Schrefl, J. Fidler, K. J. Kirk, and J. N. Chapman, J. Magn. Magn. Mater. 175, 193 (1997).

${ }^{18}$ K. J. Kirk, J. N. Chapman, and C. D. W. Wilkinson, Appl. Phys. Lett. 71, 539 (1997). 